\title{
Distinct phenotypes in COVID-19 may require distinct pulmonary rehabilitation strategies
}

\author{
Eduardo Eriko Tenório de França1, Ubiracé Elihimas Junior ${ }^{2}$, Paulo Adriano Schwingel², \\ Celso R.F. Carvalho ${ }^{3}$, Maria do Socorro Brasileiro-Santos ${ }^{1,4,5}$
}

${ }^{1}$ Department of Physiotherapy and Postgraduate Program in Physiotherapy, Federal University of Paraiba, João Pessoa; ${ }^{2}$ Postgraduate Program in Health Sciences, University of Pernambuco, Pernambuco; ${ }^{3}$ Department of Physical Therapy, University of São Paulo, São Paulo; ${ }^{4}$ Department of Physical Education, Federal University of Paraiba; ${ }^{5}$ Associate Graduate Program in Physical Education, UPE-UFPB, Brazil

\begin{abstract}
The novel coronavirus (SARS-CoV-2) has distinct clinical manifestations that can vary from an asymptomatic condition to severe acute respiratory failure. Phenotypes are attributable to different pathophysiological mechanisms and require different treatment strategies. The assessment and identification of different phenotypes can guide therapy configurations such as oxygen therapy, non-invasive ventilation, airway management, and tracheal intubation. Further studies are essential to provide information on the influence of phenotypes in the decision of rehabilitation strategies. The sequelae left in the respiratory system of COVID-19 survivors and its limitations will be a challenge for rehabilitation services worldwide. Lung injuries are directly
\end{abstract}

Correspondence: Eduardo E.T. de França, Department of Physiotherapy
and Postgraduate Program in Physiotherapy, Federal University of
Paraiba, Conj. Pres. Castelo Branco III, João Pessoa, PB, 58033-455,
Brazil.
E-mail: edueriko@hotmail.com

Key words: COVID-19; pneumonia; phenotypes; physical and rehabilitation medicine.

Authors' contributions: All authors contributed equally to the manuscript and read and approved the final version of the manuscript.

Conflicts of Interest: The authors certify there is no conflict of interest with any financial organization regarding the material discussed in the manuscript.

Funding: The authors report no involvement in the research by the sponsor that could have influenced the outcome of this work.

Received for publication: 22 July 2020.

Accepted for publication: 4 November 2020.

${ }^{\circ}$ Copyright: the Author(s), 2020

Licensee PAGEPress, Italy

Monaldi Archives for Chest Disease 2020; 90:1523

doi: 10.4081/monaldi.2020.1523

This article is distributed under the terms of the Creative Commons Attribution Noncommercial License (by-nc 4.0) which permits any noncommercial use, distribution, and reproduction in any medium, provided the original author(s) and source are credited. related to the phenotypes presented, and depending on the degree of these injuries, rehabilitation strategies can be targeted. We believe that differentiating patients, according to their respective phenotypes, can improve decision-making in treatment and individualized rehabilitation.

The novel coronavirus (SARS-CoV-2) has distinct clinical manifestations that can vary from asymptomatic to severe acute respiratory failure [1]. Imaging features of viral pneumonia associated with SARS-CoV-2 may also vary from mild opacification with near-normal radiographs to tomographic spectra such as ground-glass opacifications (83\%), ground-glass opacifications with mixed consolidation (58\%), pleural thickening (52\%), interlobular septal thickening (48\%) and air bronchograms (46\%). The clinical representation of the most severe phenotype is acute respiratory failure, which can rapidly progress to hypoxemia [1]. Critical illness with severe acute respiratory failure, shock, and multiorgan dysfunction is found amongst approximately $5 \%$ of patients [2].

Phenotypes are attributable to different pathophysiological mechanisms and require different treatment strategies. Gattinoni et al. [3] proposed a classification in two phenotypes. Phenotype "L" or "low" present low elastance, low perfusion ventilation (VA/Q), low pulmonary weight, and low lung recruitment capacity. On the other hand, phenotype "H" or "high" present high elastance, high shunt right-left, high pulmonary weight, and high pulmonary recruitment [3].

Robba et al. [4] proposed to classify different phenotypes based on computed tomographic scan (CT), to stage lung disease by COVID-19 in three phenotypes of viral pneumonia SARSCoV-2. Phenotype-1 is similar to the low phenotype proposed by Gattinoni et al. [3]. Phenotype-2 is a transitional phenotype, which reflects the evolution of the disease while phenotype-3 corresponds to the "high" Gattinoni et al. [3] phenotype.

Comorbidities, direct lung damage from COVID-19, and simultaneous injuries to other organs are important factors that need to be considered in order to elaborate a rehabilitation treatment plan for patients recovering from COVID-19. The most likely early complications are acute respiratory distress syndrome, sepsis, septic shock, multiple organ failure, acute kidney, and cardiac injuries. These complications contribute to the need for ICU admissions; however, we still do not know how long these complications last, nor their clinical repercussions [5].

Chest CT assessment is extremely important to individualize treatment and mechanical ventilation settings. In our opinion, 
given the low resolution of plain radiography, we recommend that chest CT should be performed in all critically ill patients. Typical findings of chest CT in COVID-19 include bilateral infiltrates with multiple ground-glass opacities or consolidation without oedema. Some patients also exhibit asymmetric oedematous lesions and atelectasis or dispersed fibrosis [6].

The assessment and identification of different phenotypes can guide therapy configurations such as oxygen therapy, non-invasive ventilation, airway management and tracheal intubation, prone position, inhaled nitric oxide, recruitment manoeuvres, and extracorporeal membrane oxygenation. In addition, tomographic phenotypes are associated with other clinical manifestations and disease severity like hypercoagulability, hemodynamic instability, cardiac injury, and sepsis. These manifestations can also be indirect predictors of weaning difficulty and extubation failure [4]. Further studies are essential to provide information on the influence of phenotypes in the decision of rehabilitation strategies.

Practical and viable respiratory rehabilitation guidelines for patients with COVID-19 were organized based on the consensus and references of Chinese frontline specialists. [7]. Current guidelines focus on two main targets: i) short-term goal of pulmonary rehabilitation aiming to relieve dyspnoea and anxiety and depression symptoms; ii) long-term goal to preserve the patient's general functionality, improve QoL and facilitate return to regular daily life activities [7]. Before tailoring a post-COVID-19 rehabilitation program, it is prudent to assess the risks of exercise based on clinical, laboratory, and CT exams, without forgetting to evaluate comorbidities. The identification of the phenotypes must be part of the design of the pulmonary rehabilitation program, and this classification must be included in the study protocols.

Multi-professional approach using questionnaires to evaluate health-related quality of life (HRQoL), daily activities, nursing evaluation, psychological and nutritional status, are part of a qualitative analyses instruments that must also be a part of postCOVID-19 treatment protocols [7]. The results of these assessments revolve around with the patient's aerobic capacity, muscle strength, balance, and flexibility to formulate an individualized and progressive rehabilitation prescription [7].

COVID-19 is advancing rapidly; still, health-related agencies and societies do not have evidence-based guidelines. However, there are some guidelines suggested by health professionals based on their experiences and opinions of experts [8]. Health teams must propose an individualized treatment based on case-by-case evaluation. Direct assessment of the COVID-19's impact on the respiratory, neuromuscular, immune, cardiovascular, and kidneys systems and the sequelae and rehabilitating comorbidities should be associated with the COVID-19 phenotypic manifestations to guide treatment plan [8].

The World Health Organization does not have rehabilitation guidelines and, in particular, pulmonary rehabilitation guidelines, for post-COVID-19 patients, which is justified by the scarcity of studies. To the best of our knowledge, there is only one randomized controlled study analysing respiratory, physical, and psychological capacity outcomes in elderly rehabilitates with different degrees of COVID-19 clinical expressions [9]. Pulmonary rehabilitation lasted for six weeks, and the assessment was based on respiratory function, HRQoL, mobility, and psychological aspects. There was an overall improvement in all aspects, except on patients' psychological evaluations on which depression remained [9].

We believe that not all post-COVID-19 patients will develop significant pulmonary sequelae even in those patients who have the "high" phenotype [3] or "3" phenotype [4] who needed mechanical ventilation. These patients will most benefit from pul- monary rehabilitation programs, which should be aimed at pulmonary expansion therapy, respiratory muscle training, and strengthening and aerobic exercises for upper and lower limbs.

The patient who develops the "high" phenotype will present greater pulmonary complications, will remain for longer periods of stay in ICU, develop peripheral muscle weakness, have more reduction in the functional capacity, and experience greater fatigue. Therefore, this type of patient will need a long-term follow-up in cardiorespiratory rehabilitation to facilitate muscle training and functional recovery. For this group of patients, our protocol should include the use of either non-invasive ventilation or expiratory pressure airway positive for pulmonary expansion therapy (depending on the assessment of lung function), respiratory muscle training with isokinetic inspiratory load, lower limb cycle ergometers, strengthening muscle exercises and neuromuscular electrical stimulation for improving functional capacity and recovering muscle strength in patients.

Concerning patients who presented with the other phenotypes, they may also need pulmonary physical rehabilitation; therefore, the need to identify the phenotypic manifestations to assess our patients and outline individualized rehabilitation strategies. The other phenotypes seem to present fewer impact on lung function and greater involvement in the cardiovascular system, thus these patients with "low" phenotype could directly benefit on cardiovascular rehabilitation. Our protocol has focused on cardiovascular rehabilitation using a stationary bicycle or treadmills, considering an intensity load ranging from 60 to $85 \%$ of the functional capacity obtained through the maximum heart rate assessed in a maximum exercise test and in the resistive training of limbs higher with low load and a greater number of repetitions [10].

The sequelae left in survivors of COVID-19 respiratory system and its limitations will be a challenge for rehabilitation services worldwide. Lung injuries are directly related to the phenotypes presented, and depending on the degree of these injuries, rehabilitation strategies can be targeted. We believe that differentiating patients, according to their respective phenotypes, can improve decision-making in treatment and individualized rehabilitation. However, further randomized studies are recommended to confirm the suggested rehabilitation hypotheses.

\section{References}

1. Rello J, Storti E, Belliato M, Serrano R. Clinical phenotypes of SARS-CoV-2: implications for clinicians and researchers. Eur Respir J 2020;55:2001028.

2. Bao C, Liu X, Zhang H, et al. Coronavirus disease 2019 (COVID-19) CT findings: a systematic review and metaanalysis. J Am Coll Radiol 2020;17:701-9.

3. Gattinoni L, Chiumello D, Caironi P, et al. COVID-19 pneumonia: different respiratory treatments for different phenotypes? Intensive Care Med 2020;46:1099-102.

4. Robbaa C, Battaglinia D, Balla L, et al. Distinct phenotypes require distinct respiratory management strategies in severe COVID-19. Respir Physiol Neurobiol 2020;279:103455.

5. Sheehy LM. Considerations for postacute rehabilitation for survivors of COVID-19. JMIR Public Health Surveill 2020;6:e19462.

6. Zhang JJ, Dong X, Cao YY, et al. Clinical characteristics of 140 patients infected with SARS-CoV-2 in Wuhan, China. Allergy 2020;75:1730-41.

7. Yang Lu-Lu, Yang T. Pulmonary rehabilitation for patients 
with coronavirus disease 2019 (COVID-19). Chronic Dis Transl Med 2020;6:79-86.

8. Stam HJ, Stucki G, Bickenbach J. Covid-19 and post intensive care syndrome: a call for actio. J Rehabil Med 2020;52:j rm00044.
9. Liu K, Zhangb W, Yangc Y, et al. Respiratory rehabilitation in elderly patients with COVID-19: a randomized controlled study. Complement Ther Clin Pract 2020;39:101166.

10. ACSM. ACSM guidelines for stress testing and prescription. 6th ed. Ed. Guanabara Koogan: Rio de Janeiro; 2003. 